\title{
Study on Relationship between Polar Compounds and LF-NMR Properties in Fried Camellia Seed Oil
}

\author{
Jinying Wang ${ }^{1,2, *}$, Chaosheng Liu ${ }^{2}$, Dale Sun ${ }^{2}$ \\ ${ }^{1}$ State Key Laboratory of Plateau Ecology and Agriculture, Qinghai University, Xining, P R China \\ ${ }^{2}$ College of Agriculture and Animal Husbandry, Qinghai University, Xining, P R China \\ *Corresponding author: wangjinying0128@126.com
}

\begin{abstract}
The correlation between classic column chromatography and LF-NMR to determine the total polar compounds were investigated in this study. Results revealed that the content of polar compounds in fried camellia seed oil were keeping elevated during deep frying and reach to reject point up to $24 \mathrm{~h}$, the content level were $27.21 \%$. The significant correlation was existed between TPC and S21, LF-NMR could replace the classic column chromatography as the rapid detection way to determine the TPC in fried oils.
\end{abstract}

Keywords: fried camellia seed oil, LF-NMR, polar compounds

Cite This Article: Jinying Wang, Chaosheng Liu, and Dale Sun, "Study on Relationship between Polar Compounds in Fried Camellia Seed Oil and LF-NMR Properties." Journal of Food and Nutrition Research, vol. 6, no. 7 (2018): 433-438. doi: 10.12691/jfnr-6-7-2.

\section{Introduction}

Camellia oleifera Abel., an evergreen tree, grows only in middle and southern regions of China, such as Hunan, Jiangxi, Fujian, Anhui, Guangdong provinces, etc. [1]. The world's total plant area of camellia is around 3.3 million ha, of which approximately 3.0 million ha in China standing about $90 \%$ of the global total. In addition to Southeast Asia, Japan and other State-owned very little distribution $[2,3]$. Camellia oleifera is considered to be nutritious and healthy, owing to its fatty acids composition and the presence of bioactive compounds, such as tea polyphenol, tea saponin and squalene, etc. [4]. Camellia oil (CO) is obtained from Camellia oleifera seeds, is characterized by the high proportion of oleic acid (74-89\%) [5]. CO has been used as a cooking oil in China for 2300 years [9]. It is characterized by a high proportion of oleic acid (74-89\%) [10] and antioxidant compounds, such as sterols, triterpenes [11], tocopherol, squalene [12] and phenolics $[13,14]$.

Polar compounds were resulted from oxidation, polymerization, splitting decomposition and hydrolization during thermal process of edible oils, which exhibits higher polarity than normal vegetable oil molecules [15]. Because of total polar compounds represent the all decomposition product exist in samples, it can be used as good index of degradation of fried oils [16]. Polar compounds had detrimental effect to the man's health. Ames experiment and bone marrow micronucleus rate test conducted by Liu et al. [17] demonstrate that fried oils and polar compounds showed mutagenic effects in some extent. Therefore, in order to control the oil quality applied in the manufacturing industry and experiment process, many countries set the maximum limit for total polar compounds level in fried oils. Such as, Australia and China were 27\%, Belgium, France and Spain were 25\%, Germany were $24 \%$.

The determination of polar compounds including two aspects, namely determine the levels of total polar compounds and analysis the constitution of them. As the most reliable and widely used method to determine the total polar compounds in fried oils, column chromatography was the standard method approved by IUPAC [18] and AOCS [19]. It is also the standard method in China. Economic and effective micro-column chromatography [20] and Solid-phase extraction (SPE) [21] were derived from classic column chromatography. After separate the polar compounds by method to determine the total polar compounds and then introduce the fraction onto the highperformance size exclusion (HPSEC). As result of the combined separation criteria used, firstly based on polarity and secondly on molecular size or weight, five groups of compounds are neatly separated and quantified: TG oligomers (TGO), TG dimers (TGD), oxidized TG monomers (ox-TGM), diacylglycerols (DG), and free fatty acids (FFA), in this order of elution [20] [22].

In recent years, the environmental and nondestructive spectrometry method had overtaken the absorption chromatography become the main measure to determine the polar compounds in fried oil rapidly. LF-NMR (Lowfield nuclear magnetic resonance) was one of them. LFNMR, a relaxation spectrum analysis technology based on nuclear magnetic [23], which could detect the physical and chemical information and unique fingerprint database of proton or nucleon of sample simultaneously [24]. As a new type of rapid detection technology, LF-NMR was play an import role in the analysis of fried oil quality [25]. According to Wang et al. [26], there was a significant correlation between S21 in transverse relaxation spectrum and content level of total polar compounds. This theory 
was combined with LF-NMR and applied in predict the level of total polar compounds in fried oils by Yang et al. [27]. A number of study focused on the oil quality evaluation by LF-NMR indicate that LF-NMR no only used for oil adulteration $[28,29]$, meanwhile, applied in reflect the quality of fried oils effectively and were not affected by antioxidant [30,31,32].

Up to date, there is no report on the use of LF-NMR as a rapid detection technology for analysis of Camellia seed oils. Therefore, the objective of this study was to investigate the correlation between chemical process (classic column chromatography) and spectrometry method (Low-field nuclear magnetic resonance, LF-NMR) to determine the level of total polar compounds.

\section{Materials and Methods}

\subsection{Materials}

The crude CO were collected in Dong Lan where located in Guang Xi Province in China. The refined CO were purchased from local market. The n-hexane, Petroleum ether (b.p. $30-60^{\circ} \mathrm{C}$ ), diethyl ether, silica gel - particle size $0.063-0.200 \mathrm{~mm}$ (70-230 mesh) and sea sand were all acquired from Sinopharm Chemical Reagent Co., Ltd in ShangHai.

\subsection{Frying Protocol}

Frying experiments were carried out using 8 L capacity domestic deep-fat electric fryer (HY-81, GangYang electromechanical equipment company) equipped with a removable stainless steel wire basket and a thermostat. Fresh potato was shelled shortly before frying and cut into chips (approximately 40-50×10×10 mm), which needed rinsed with cold water and drained for a while prior to being submerged into the $4 \mathrm{~L}$ sample $\mathrm{G}$. To avoid temperature losses in the oil during frying, small amounts of potatoes (125 g) were fried each time. A batch of potato chips was fried for $4 \mathrm{~min}$ at $11 \mathrm{~min}$ intervals for a period of $6 \mathrm{~h}$ per day with 24 batches fried per day for 7 consecutive days. Frying oil sample (50 g) was withdrawn in amber bottles at $2 \mathrm{~h}$ intervals and after cooling to room temperature stored at $-20^{\circ} \mathrm{C}$ until further analysis. During the frying period, the fryer was left uncovered. After each frying operation, the fryer was switched off and the temperature was allowed to drop to $60^{\circ} \mathrm{C}$ until the next session. No fresh oil was added for replacement of the oil removed during sampling. The fryers were then cupped with their lids and the remaining oil was allowed to cool overnight. For the first batch of each day frying test, the oil was needed conditioned by heating to $185 \pm 50^{\circ} \mathrm{C}$ and held for $30 \mathrm{~min}$.

\subsection{Determination of Total Polar Compounds}

According to the standard method of China [15] and AOCS [20], 2.5 $\pm 0.1 \mathrm{~g}$ of used frying oil or fat dissolved in the $10 \mathrm{~mL}$ elution solvent is introduced onto a glass column filled with a slurry of silica gel and elution solvent. A chromatographic glass column of $(2 \mathrm{~cm} \times 40 \mathrm{~cm})$, and
$25 \mathrm{~g}$ of silica gel (70-230 mesh) - adjusted to a water content of $5 \%$ are used. The elution solvent is a mixture of light petroleum (b.p. $30-60^{\circ} \mathrm{C}$ ) and diethyl ether 87:13 (V/V). Elution of non-polar compounds, including mostly unoxidized TG, is carried out with $150 \mathrm{~mL}$ of the elution solvent and then the polar compound fraction is eluted with $150 \mathrm{~mL}$ of diethyl ether. A dropping funnel is used and the flow rate is adjusted to about $2.5 \mathrm{~mL} / \mathrm{min}$. Fractions are collected in round bottom flasks and solvents are removed with the aid of a rotary evaporator. Fractions can be dried to constant weight using a stream of nitrogen. The mass fractions of non-polar compounds $\left(\mathrm{w}_{\mathrm{np}}\right)$ and the polar compounds $\left(\mathrm{w}_{\mathrm{p}}\right)$ are given by the formula:

$$
\begin{gathered}
W_{n p} \%=\frac{m_{n p}}{\mathrm{~m}} \times 100 \\
W_{p} \%=\frac{m_{p}}{\mathrm{~m}} \times 100
\end{gathered}
$$

where $m_{n p}$ is the mass (in g) of the nonpolar fraction $1, m_{p}$ is the mass (in $\mathrm{g}$ ) of the polar fraction 2 , and $\mathrm{m}$ is the mass (in $\mathrm{g}$ ) of the test portion added to the column

The efficacy of the separation is assessed by thin-layer chromatography (TLC). Glass plates coated with silica gel, $0.25 \mathrm{~mm}$ layer, and a mixture of light petroleum, diethyl ether and acetic acid 70:30:2 (V/V/V) as developing solvent are used. After plate development and solvent evaporation, the plate is sprayed with a phosphomolybdic acid solution and heated at $120-130^{\circ} \mathrm{C}$ to visible spots. The efficacy of the separation of polar compounds and nonpolar compounds were estimated by retardation factor (Rf).

\subsection{Determination of Transverse Relaxation Time}

Transverse relaxation time (T2) were determined by CPMG (Carr-Purce II -Meiboom-Gill) impulse sequence, the temperature was set at $32 \pm 0.01^{\circ} \mathrm{C}$. Weight $2.0 \mathrm{~g}$ fried Camellia seed oil accurately put in the nuclear magnetic resonance exclusive test tube (diameter, $15 \mathrm{~mm}$ ). After maintain constant temperature at $32 \pm 0.01^{\circ} \mathrm{C}$ in Water-bathing 10 min place the tube in the nuclear magnetic resonance probe, stabilize $1 \mathrm{~min}$ and then start data collection. When finish the collecting, place the sample in Water-bathing 5 min main constant temperature at $32 \pm 0.01^{\circ} \mathrm{C}$ reassembly so as to next determination. Equipment parameter settings were as follows: Repeating Time (TR) $1500 \mathrm{~ms}$, half-echo time $(\tau) 250 \mu \mathrm{s}$, Echo Count 8000, sampling numbers (TD) 754038, scanning number (NS) 4 times, spectral width (SW) $250 \mathrm{kHz}$. Each sample repeat sampling 6 times.

\subsection{Statistical Analysis}

Each experiment was done in triplicate. The results were expressed as mean \pm standard deviation and were analyzed by SPSS (version 17.0 SPSS Inc., USA). Oneway analysis of variance was performed using ANOVA procedures and regression. Significant differences between the means were determined by Duncan's Multiple Range test. $P<0.05$ was considered as a level of significance. 


\section{Results and Discussion}

\subsection{Optimization of Major LF-NMR Parameters}

\subsubsection{Repetition Time (TR)}

Repetition Time (TR) were the duration of prior sampling to last sampling. Different samples were characterized by different TR, which were related to the energy release speed of them. The magnitude of TR was determined by the longest relaxation time.

As showed in Figure 1, The maximum signal mode of sampling signal was tended to become stable gradually with the enlarge of TR value. It is difficult to maintain a balanced state when the TR value were too small. When TR value reach to $1500 \mathrm{~ms}$ the signal modulus value of each sampling was relatively stable, which means that the selected TR value can enable the sample system return to equilibrium state. Along with the further increasing of TR value up to $2000 \mathrm{~ms}$, the stability of signal collected from sampling were reduced. The greater value of TR were set, the longer waiting time of sampling were acquired and lead to the reduction of experiment effective. Therefore, select the $1500 \mathrm{~ms}$ as the optimistic parameter of TR.

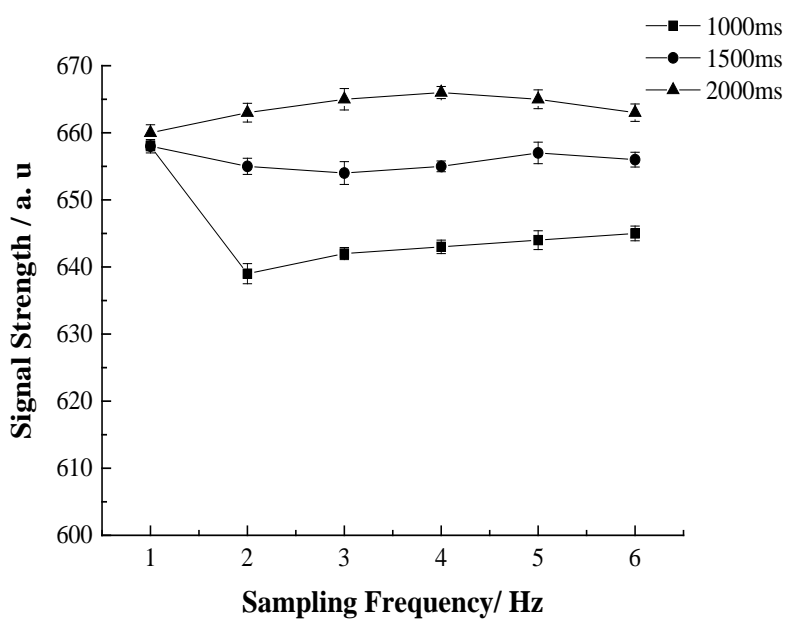

Figure 1. The effect of TR on the stability of LF-NMR detect signal

\subsubsection{Half-echo Time $(\tau)$}

Half-echo time were the time interval between 90 degree pulse and adjacent 180 degree pulse. The change of $\tau$ value may affect the shape of signal, which were resulted from the intra-molecular free diffusion and molecule exchange of sample. Select the minor $\tau$ value if there is a need to narrow down the effect of intra-molecular free diffusion on signal, otherwise choose the major one. As show in Table 1, when $\tau$ set at $150 \mu$ s, the distributed scope T2 value in acquired curve of CPMG sequence were $0-1500 \mathrm{~ms}$ and signal amplitude in vertical axis were attenuated from maximum to 12 . With the increase of $\tau$ value the distribution interval of T2 were enlarged gradually. The attenuation in signal amplitude of corresponding echo curve demonstrate the completeness of echo curve. When set the $\tau$ value at $250 \mu$ s, signal amplitude was attenuated to minimum and meet the determination requirements for $\mathrm{CO}$.
Table 1. Effect of $\tau$ value on the CPMG echo decay curve

\begin{tabular}{ccc}
\hline$\tau / \mu \mathrm{s}$ & T2/ms & SP/a.u \\
\hline 150 & 1000 & 11.603 \\
200 & 1500 & 3.43 \\
250 & 2000 & 1.217 \\
\hline
\end{tabular}

Note: $\tau$ - Half echo time; T2-Transverse relaxation time; SP-Signal modulus value.

\subsubsection{Half-echo Time $(\tau)$}

Echo count were the echo number after the signal collection. In general, enough echo count was needed to relax the signal completely. With the increase of echo count the distribution interval of $\mathrm{T} 2$ were enlarged and signal amplitude of corresponding echo curve were attenuated gradually. As show in Table 2, Signal amplitude attenuated to 8 when the echo count was 4000 , which indicate that echo count was too small to attenuate the signal amplitude completely. Along with the echo count reach to 6000 and 8000 , the signal amplitude was attenuated to 2 and 1 respectively. Finally, select the 8000 as the most suitable echo count.

Table 2. Effect of $\tau$ value and EchoCount on the CPMG echo decay curve

\begin{tabular}{ccc}
\hline EchoCount & T2/ms & SP/a.u \\
\hline 4000 & 1600 & 9.011 \\
6000 & 2400 & 2.034 \\
8000 & 3200 & 1.008 \\
\hline
\end{tabular}

Note: T2-Transverse relaxation time; SP-Signal modulus value.

\subsection{The Correlation between Total Polar Compounds (TPC) and LF-NMR Data}

The determination of TPC indicate that the content of TPC in fresh CO were $4.58 \%$, with the prolonged duration the level of polar compounds in fried CO were keeping elevated and reach to reject point up to $24 \mathrm{~h}$. At the end of frying process, the content climbed to $42.49 \%$. The accumulation of TPC and frying time were significantly correlated during whole frying process, this finding was accordance with the research of Liang et al. [33].

Figure 2 were the typical T2 spectrum of fresh and fried CO, three peaks were successively marked as T21, T22 and T23, the area of each peak were represented as S21, S22 and S23 respectively. As can be seen from Figure. 2, the $\mathrm{T} 2$ spectrum of fresh refined $\mathrm{CO}$ were consisted by T22 and T23, while a new peak was generated in fried oil, Wang et al. [32] reported similar results. As show in Table 3, the S21 were increased with the extend of frying time, while the S22 and S23 present a decreased tendency. The decrease rate of S23 were 2 times of S22, this could be ascribed to the quicker degradation of unsaturated fatty acid, because generally S22 and S23 in typical T2 spectrum were represent the level of saturated and unsaturated fatty acid respectively. The S21, area of new oxidized compounds differentiate from Triglyceride composition, were increased during frying process and present significant correlation with frying time $\left(y=2.7516 x+10.605, R^{2}=0.9717\right)$. The content increase of compounds characterized by similar valve of T21 strengthen the signal response of LF-NMR 
and the result the heighten of the corresponding area (S21) consequently [32,34,35].

Besides, the starting time of T21, T22 and T23 were all shrink with the increase of frying time and showed good correlation with it $\left(\mathrm{R}^{2}>0.96\right)$. Otherwise, research focused on soybean oils attested that there was no obvious regular changes of starting time of T23 [30], this difference could be attributed to the oxidation and per-oxidation product generated from complex chemical reaction, such as oxidation, splitting and hydrolysis. Xiong Guohuan [36] and Wang Yongwei [32] maintains that the shorten of transverse relaxation time were closely associated with compounds that generated in frying process and characterized by large size, complex construction, long chain and huge molecular, which will generate bucking effect resulted from nuclear spin of local magnetic field by increase the overall intermolecular interaction force and binding effect. Finally, results in the shorten of transverse relaxation time. In addition, the polymerization degree of oxidation products that produced during frying were enlarged with the prolonged process and the relaxation of hydrogen proton in magnetic field were affected by intermolecular interaction, ultimately resulting in a overall migration of T2 spectrum [26].

The content of total polar compounds (TPC) and S21 were correlated closely with frying time and Fan Zhixiong [37] insist that S21 in T2 spectrum could reflect the quality of fried oils without the effect of antioxidant [31]. Hence, the LF-NMR technology could be applied in rapid detection of edible oils effectively [26,30]. As depicted in Figure 3, the greater correlation was existed between total polar compounds (TPC) and S21, which in accordance with the finding of Shen et al. [38]. LF-NMR analysis technology that depended on this theory were applied in predict the total polar compounds in fried oils, which means that LF-NMR analysis technology based on spectroscopy could replaced the classic column chromatography based on chemical method as the rapid detection way to determine the total polar compounds in fried oils.

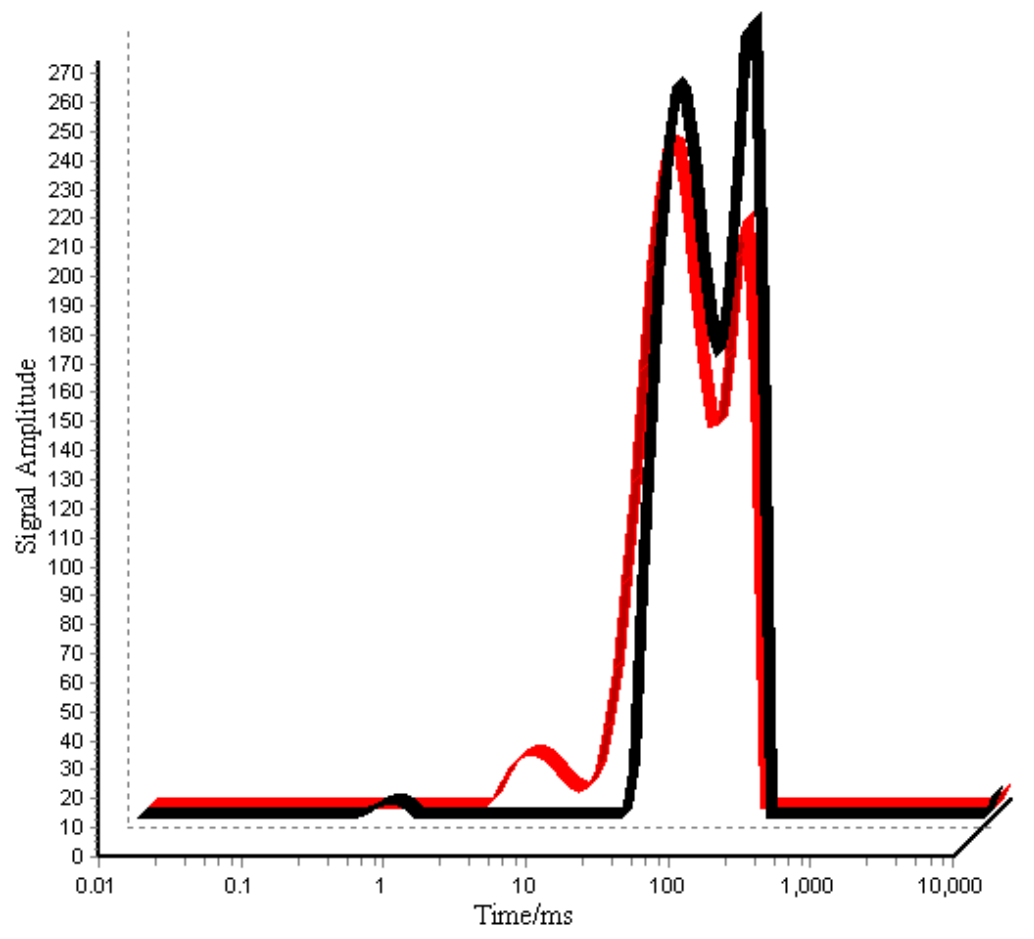

Figure 2. Typical transverse relaxation time(T2) spectrum of Camellia seed oil

Table 3. Change of Starting Time of peak and character peaks area of fried Camellia seed oil $(n=6)$

\begin{tabular}{|c|c|c|c|c|c|c|}
\hline \multirow{2}{*}{ Time/h } & \multicolumn{3}{|c|}{ Starting Time of peak/ms } & \multicolumn{3}{|c|}{ Character Peaks Area } \\
\hline & $\overline{\mathrm{T} 21}$ & T22 & $\overline{\mathrm{T}} 23$ & $\mathrm{~S} 21$ & S22 & S23 \\
\hline 0 & $5.21 \pm 0.12$ & $26.63 \pm 0.98$ & $157.68 \pm 0.65$ & $18.27 \pm 0.34$ & $2165.59 \pm 3.54$ & $915.13 \pm 2.01$ \\
\hline 4 & $5.01 \pm 0.28$ & $23.16 \pm 0.45$ & $153.73 \pm 0.76$ & $25.68 \pm 0.38$ & $2137.33 \pm 2.78$ & $897.74 \pm 2.45$ \\
\hline 8 & $4.71 \pm 0.03$ & $22.04 \pm 0.36$ & $150.87 \pm 1.05$ & $33.70 \pm 0.76$ & $2124.92 \pm 4.02$ & $866.37 \pm 3.01$ \\
\hline 12 & $4.49 \pm 0.45$ & $21.16 \pm 0.45$ & $147.78 \pm 0.87$ & $37.00 \pm 0.56$ & $2079.28 \pm 3.64$ & $837.7 \pm 2.93$ \\
\hline 16 & $4.44 \pm 0.11$ & $20.61 \pm 0.76$ & $144.39 \pm 2.03$ & $54.17 \pm 0.50$ & $2054.78 \pm 3.97$ & $825.06 \pm 1.99$ \\
\hline 20 & $4.29 \pm 0.43$ & $19.64 \pm 0.65$ & $142.79 \pm 1.05$ & $59.87 \pm 0.08$ & $2056.28 \pm 2.23$ & $798.81 \pm 3.45$ \\
\hline 24 & $4.10 \pm 0.32$ & $18.74 \pm 0.34$ & $140.31 \pm 1.05$ & $66.01 \pm 0.36$ & $2046.07 \pm 3.06$ & $765.57 \pm 1.67$ \\
\hline 28 & $4.04 \pm 0.18$ & $17.74 \pm 0.45$ & $139.99 \pm 0.97$ & $85.31 \pm 0.88$ & $2045.31 \pm 2.09$ & $744.03 \pm 2.57$ \\
\hline 32 & $3.77 \pm 0.36$ & $16.30 \pm 0.56$ & $136.69 \pm 0.67$ & $96.99 \pm 0.94$ & $2034.85 \pm 3.78$ & $718.63 \pm 2.09$ \\
\hline 36 & $3.36 \pm 0.22$ & $14.17 \pm 0.56$ & $133.98 \pm 1.65$ & $118.6 \pm 0.99$ & $2020.94 \pm 3.67$ & $699.16 \pm 1.67$ \\
\hline 40 & $3.01 \pm 0.06$ & $12.17 \pm 0.76$ & $129.19 \pm 2.06$ & $126.41 \pm 1.05$ & $2001.51 \pm 4.02$ & $657.1 \pm 1.01$ \\
\hline
\end{tabular}




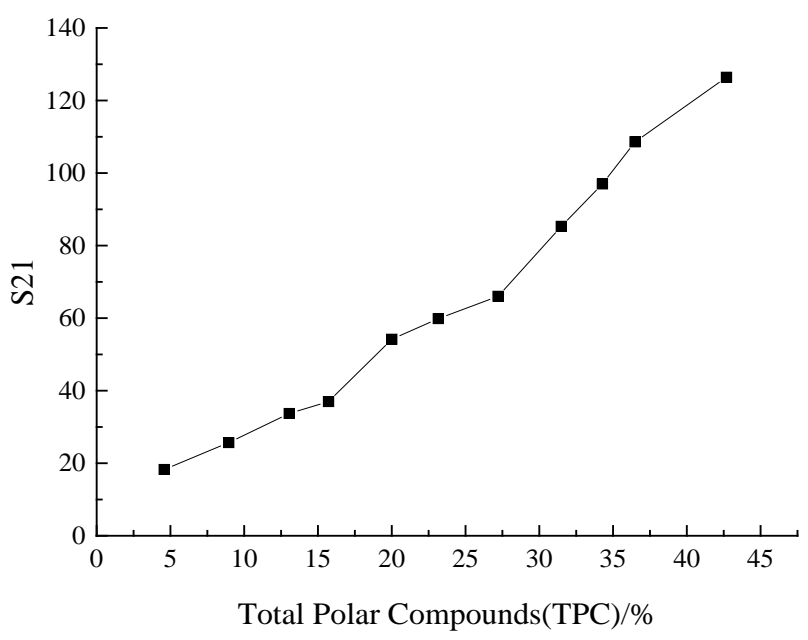

Figure 3. The correlation between total polar compounds (TPC) and S21

\section{Conclusion}

1). The optimistic parameters of LF-NMR determine the T2 of CO were: TR $=1500 \mathrm{~ms}, \tau=250 \mu \mathrm{s}$, EchoCount $=8000$, NS $=4$ times, $\mathrm{TD}=754038$, $\mathrm{SW}=250 \mathrm{kHz}$.

2 ). The content of polar compounds in fried $\mathrm{CO}$ were keeping elevated during deep frying and reach to reject point up to $24 \mathrm{~h}$. S21 in LF-NMR T2 spectrum were increased with the prolonged duration of frying, otherwise, S22 and S23 were decreased. The start time of T21, T22 and T23 were all shorted. The greater correlation was existed between TPC and S21, LF-NMR could replace the classic column chromatography as the rapid detection way to determine the TPC in fried oils.

\section{Acknowledgements}

The authors would like to thank from Key Laboratory of economic forest breeding and cultivation State Forestry Bureau, Central South University of Forestry and Technology, Changsha, Hunan, P R China, for their important experiment platform support in the research project.

\section{Foundation}

The work was funded by the Youth Nature Science Foundation of Qinghai Province, P R China (2018-ZJ-970Q).

\section{Statement of Competing Interests}

The authors have no competing interests

\section{References}

[1] Ye, Y., Guo, Y., Luo, Y. T. and Wang, Y. F. "Isolation and free radical scavenging activities of a novel biflavonoid from the shells of Camellia oleifera Abel.,” Fitoterapia, 83(8). 1585-1589. 2012.

[2] Zhang, S. and $\mathrm{Li}, \mathrm{X}$. Z, "Hypoglycemic activity in vitro of polysaccharides from Camellia oleifera Abel. seed cake," International journal of biological macromolecules, Accepted. 2018.
[3] Jin, X, "Bioactivities of water-soluble polysaccharides from fruit shell of Camellia oleifera Abel: Antitumor and antioxidant activities," Carbohydrate polymers, 87(3). 2198-2201. 2012.

[4] Wu, H., Li, C., Li, Z., Liu, R., Zhang, A., Xiao, Z. and Deng, S, "Simultaneous extraction of oil and tea saponin from Camellia oleifera Abel. seeds under subcritical water conditions," Fuel Processing Technology. 174. 88-94. 2018.

[5] Guo, L. X., Xu, X. M., Yuan, J. P., Wu, C. F. and Wang, J. H, "Characterization and authentication of significant Chinese edible oilseed oils by stable carbon isotope analysis," Journal of the American Oil Chemists' Society, 87(8). 839-848. 2010.

[6] Zhu, B., Zhong, H. Y., Cao, Q. M. and Long, Q. Z, "Advance in research on bioactive compounds in Camellia spp," Non-wood forest research, 28. 140-145. 2010.

[7] Zhang, D. S., Jing, Q. Z., Wang, X. G. and Xue, Y. L, "Research status of nutrition quality of camellia oleifera seed and oil," Science and Technology of Cereals,Oils and Foods, 21. 53-56. 2013.

[8] Wang, J. Y. and Zhong, H.Y, "Determination of the content of phenols in Camellia oil by RP-HPSEC with internal standard method," Journal of the chinese cereals and oils association, 29. 107-111. 2014.

[9] Wang, J. Y., Zhong, H. Y., Zhu, X. Y. and Zhou, B, "Study on antioxidant activity of polyphenolic compounds from Camellia polydonata seeds," Food and Machinery, 29. 105-107. 2013.

[10] Machado, E. R., Marmesat, S., Abrantes, S. and Dobarganes, C, "Uncontrolled variables in frying studies: differences in repeatability between thermoxidation and frying experiments," Grasas y aceites, 58(3). 283-288. 2007.

[11] Ruiz-Samblás, C., González-Casado, A., Cuadros-Rodríguez, L. and García, F. R, "Application of selected ion monitoring to the analysis of triacylglycerols in olive oil by high temperature-gas chromatography/mass spectrometry,” Talanta, 82(1). 255-260. 2010.

[12] Lerma-García, M. J., Vergara-Barberán, M., Herrero-Martínez, J. M., and Simó-Alfonso, E. F, "Acrylate ester-based monolithic columns for capillary electrochromatography separation of triacylglycerols in vegetable oils," Journal of Chromatography A, 1218(42). 7528-7533. 2011.

[13] Fan, L., Zhou, Y. L., Huo, Q. G., Zhu, T. H., Wang, C. X., Zhao, Z. H. and Chen, P. Y, C, "Identification of Seven Kinds of Vegetable Oils and Fats by Triacylglycerol Analysis and Principal Component Analysis," Journal of Henan University of Technology (Natural Science Edition), 35.1-5. 2014.

[14] Ruiz-Samblás, C., Cuadros-Rodríguez, L., González-Casado, A., García, F. D. P. R., de la Mata-Espinosa, P. and Bosque-Sendra, J. M, "Multivariate analysis of HT/GC-(IT) MS chromatographic profiles of triacylglycerol for classification of olive oil varieties," Analytical and bioanalytical chemistry, 399(6). 2093-2103. 2011.

[15] GB/T 5009.202: Determination of polar compouds in edible vegetable oils used in frying food, China Standards Press. 2003.

[16] Márquez-Ruiz, G. Formation of New Compounds during FryingGeneral Observations, AOCS Lipid library. 2009.

[17] Liu, Y.F., Mu Z., Shan, L., Fan, L.P. and Wang, X.G. "Mutagenicity of Frying Oil and its Polar Components Formed in Heating," Journal of the Chinese Cereals and Oils Association, 6(25).51-55. 2010

[18] IUPAC standard method 2.507: Determination of polar compounds in frying fats. In: standard method for the analysis of oil, fats and derivatives, 7th ed. (ed. International Union of Pure and applied chemistry, blackwell Oxford).1987.

[19] Márquez-Ruiz, G. Determination of polar compounds in used frying oils and fats by adsorption chromatography. AOCS lipid library. 2009

[20] Dobarganes, M.C., Velasco, J. and Dieffenbacher, A "Determination of polar compounds, polymerized and oxidized triacylglycerols, and diacylglycerols in oils and fats: results of collaborative studies and the standardized method (Technical report)," Pure and Applied Chemistry, 72(8).1563-1575.2000.

[21] Schulte, E. "Micromethod for the gravimetric determination of polar components in frying fats with ready for use columns," European journal of lipid science and technology, 102(8-9). 574-579.2000.

[22] Márquez-Ruiz, G. Frying oil: Determination of Oxidized Monomeric, Dimeric and Oligomeric Triacylglycerols; Diacylglycerols and Free Fatty Acids, AOCS Lipid library. 2009.

[23] Pedersen, H.T., Munck, L. and Engelsen, S.B., "Low-field $1 \mathrm{H}$ nuclear magnetic resonance and chemometrics combined for 
simultaneous determination of water, oil, and protein contents in oilseeds," Journal of the American Oil Chemists' Society, 77(10). 1069-1077. 2000.

[24] Hills, B., “Applications of low-field NMR to food science," Annual reports on NMR spectroscopy, 58. 177-230. 2006.

[25] Shao, X. l., Song, W. and Li, Y. F., "Research process of low-field nuclear magnetic resonance (LF-NMR) detection technology in grain and oil food," Journal of the Chinese Cereals and Oils Association, 28(7). 114-118. 2013.

[26] Wang, X., Xia, Y.M., Shi, R., Liu, B.L., "Quality Prediction of Frying Oil based on LF-NMR Relaxation Characteristics and Principal Component Regression," Journal of Chinese Institute of Food Science and Technology, 15(4). 155-163. 2015.

[27] Yang, X.P., Liu, B.L., Wang, X., Lu, H.Y. and Zhao, T.T. "Quantitative Modeling Method for Predicting Total Polar Compound Contents in Frying Oil Based on LF-NMR Relaxation Parameters,” Food Science, 35(24). 110-114. 2014.

[28] Zhao, T.T., Wang, X., Lu, H.Y. and Liu, B.L. "The Quality Assessment of Edible Oils and Fats by LF-NMR Coupled with PCA," Modern Food Science and Technology, 30(9). 179185.2014.

[29] Zhou, N, Liu, B.L., Wang, X., Wang, H.Z., Yang, P.Q., Zhou, H. "Discrimination of Edible Vegetable Oil Adulterated with Rice Bran Crude Oil by Low-field Nuclear Magnetic Resonance,” Food and Fermentation Industries, 30(9). 179-185. 2011.

[30] Shi, R., Wang, X., Liu, B.L., Lu, H.Y. and Zhao, T.T. "Relationship between Analytical Indicators of Soybean Oil and LF-NMR Characteristics During Frying Process," Journal of Instrumental Analysis, 32(6). 653-660. 2013.
[31] Zhang, Y., Liu, R.J., Jing, Q.Z. and Wang, X.G., “Application of low-filed nuclear magnetic resonance to analyze frying soybean oil quality," Journal of the Chinese Cereals and Oils Association, 29(9). 115-119. 2014.

[32] Wang, Y.W., Wang, X., Liu, B.L., Shi, R. and Yang, P.Q., "Application of Low-Field Nuclear Magnetic Resonance (LF-NMR) to Analyze Frying Oil Quality,” Food Science, 33(06). 171-175. 2012.

[33] Liang, Y.M., Zhou, B., Wang, J.Y. and Zhong, H.Y., "Effect of biophenol on oxidative stability of Camellia oil by Rancimat method," Food and Machinery, 30(6). 54-58. 2014.

[34] Zhang, Q., Saleh, A.S. and Shen, Q., "Discrimination of edible vegetable oil adulteration with used frying oil by low field nuclear magnetic resonance,” Food and Bioprocess Technology, 6(9). 2562-2570. 2013.

[35] de Oliveira Ramos, P.F., de Toledo, I.B., Nogueira, C.M., Novotny, E.H., Vieira, A.J.M. and de Vasconcellos Azeredo, R.B., "Low field 1H NMR relaxometry and multivariate data analysis in crude oil viscosity prediction," Chemometrics and Intelligent Laboratory Systems, 99(2). 121-126. 2009.

[36] Xiong, G.H. and Li, B. "Nuclear magnetic resonance (NMR) imaging principle,” Bei Jing Science Press. 2007.

[37] Fan, Z.X. and FAN, L.P. "The quality analysis of frying palm oil based on dielectric constant and low-field nuclear magnetic," Science and Technology of Food Industry, 33(15). 74-786. 2012.

[38] Wang, Y.W., Wang, X., Liu, B.L., Shi, R. and Yang, P.Q., "Application of Low-Field Nuclear Magnetic Resonance (LF-NMR) to Analyze Frying Oil Quality,” Food Science, 33(06): 171-175. 2012. 